\section{Cureus}

Received 01/10/2014

Review began 01/11/2014

Published 01/29/2014

\section{(c) Copyright 2014}

Nezhat et al. This is an open access article distributed under the terms of the Creative Commons Attribution License CC-BY 3.0., which permits unrestricted use, distribution, and reproduction in any medium, provided the original author and source are credited.

\title{
Use of Laparoscopic Modified Nerve- Sparing Radical Hysterectomy for the Treatment of Extensive Endometriosis
}

\author{
Camran Nezhat ${ }^{1}$, James Xie ${ }^{2}$, Diana Aldape ${ }^{3}$, Louise P. King ${ }^{4}$, Rugeen Rose Soliemannjad ${ }^{3}$, \\ Erika Balassiano ${ }^{3}$, Farr Nezhat ${ }^{5}$ \\ 1. Center For Special Minimally Invasive Surgery, Stanford University School of Medicine 2. Stanford \\ University School of Medicine 3. Center for Special Minimally Invasive and Robotic Surgery, Stanford \\ University School of Medicine 4. BIDMC, Harvard Medical School 5. St. Luke's and Roosevelt Hospitals, \\ St. Luke's and Roosevelt Hospitals
}

$\square$ Corresponding author: Camran Nezhat, nezhatinstitute@gmail.com Disclosures can be found in Additional Information at the end of the article

\section{Abstract}

INTRODUCTION: Endometriosis is an estrogen-dependent chronic inflammatory condition affecting 6-10\% of reproductive-aged women. Chronic pelvic pain impacts the quality of life of patients with endometriosis. Here, we describe the use of laparoscopic modified radical hysterectomy for the treatment of extensive and deeply infiltrating endometriosis.

METHODS: A retrospective chart review was conducted on patients with Stage IV endometriosis who underwent laparoscopic modified radical hysterectomy. All patients had a history of extensive endometriosis that failed medical and conservative surgical treatment and caused significant recurrent symptoms. The objective of this study is to show the feasibility of treatment of Stage IV endometriosis by laparoscopic modified radical hysterectomy.

RESULTS: A total of 52 patients underwent laparoscopic modified nerve-sparing radical hysterectomy for endometriosis between October 2006 and September 2013. The most common preoperative symptom was chronic pelvic pain. Adjunctive procedures, including salpingooophorectomy, appendectomy, disc excision of the bowel and ureteroneocystostomy, and in one case bowel resection, were performed as indicated. Mean patient age was 44 years (range 32-55). Mean hospital stay was one day (range 0-3). Postoperative complications included one case of urinary retention, one vaginal cuff abscess, and one infected ureteral stent. Mean follow-up was 33 months (range 13-65). Out of 52 patients, 33 had at least one prior surgery for the treatment of endometriosis. All patients reported dramatic pain relief, and there were no reports of symptom recurrence.

CONCLUSION: In cases of severe endometriosis, the use of laparoscopic modified nervesparing radical hysterectomy is a feasible and effective method for achieving long-term improvement in pain symptoms. There was no difference noted between symptom recurrences in the group of patients with bilateral salpingo-oophorectomy versus those with ovarian conservation in contrast to prior studies. Of note, 14 patients underwent bilateral oophorectomy, nine patients underwent unilateral oophorectomy, and two patients underwent removal of ovarian remnant.

Categories: Obstetrics/Gynecology, General Surgery

Keywords: endometriosis, laparoscopic hysterectomy, chronic pelvic pain, radical hysterectomy, 


\section{Introduction}

Endometriosis affects 6-10\% of reproductive-aged women [1]. The quality of life of patients with severe endometriosis can be greatly limited, most commonly by symptoms of pelvic and abdominal pain. Management of endometriosis includes medical, surgical, and combinations of both medical and surgical therapy. Medical therapies include gonadotropin-releasing hormone, progestins, estrogen-progestin combination pills, and aromatase inhibitors. The use of these medications in conjunction with conservative ablative laparoscopic treatments has become routine for pelvic pain secondary to endometriosis. When endometriosis is treated with conservative surgery, approximately $25 \%$ of patients will require subsequent surgery due to recurrent endometriosis or progression of microscopic disease [2].

Severe endometriosis and infiltrating lesions of the pelvis, intestine, and genitourinary tract can cause debilitating pain and even secondary problems, such as bowel and ureteral obliteration and end-organ damage. This may lead to more radical treatment in the form of extirpative surgical procedures.

There is no consensus on the optimal surgical management of severe endometriosis. Several authors have described radical surgery by laparoscopic excision of all detected endometriosis, while conserving non-involved structures to achieve improvement in quality of life [3-4]. This technique can often require an extensive laparoscopic "en bloc" resection of the area between the uterosacral ligaments, rectovaginal septum, and anterior rectum [5].

After a thorough literature search, only one study was found which investigated the role of radical excision of deep endometriosis, the result of which was encouraging [6]. The prospective study consists of 57 consecutive patients who underwent radical laparoscopic excision of deep endometriosis. These patients answered a questionnaire preoperatively and four months postoperatively related to their pain and quality of life. In this study, patients with radical laparoscopic excision of endometriosis had significant pain relief postoperatively. This report represents the first description of the role of laparoscopic radical excision of deep endometriosis.

\section{Materials And Methods}

A retrospective chart review was conducted on patients with Stage IV endometriosis who underwent laparoscopic modified radical hysterectomy between October 2006 and September 2013. All patients had a history of extensive endometriosis, had failed medical or surgical treatment, and had significant recurrent symptoms, such as pelvic and abdominal pain. Six of these patients underwent robot-assisted laparoscopic radical hysterectomy and the rest had standard laparoscopic radical hysterectomy. Out of 52 patients, 33 had at least one prior surgery (range: one--five) for the treatment of endometriosis.

All patients received standard outpatient preoperative evaluation, perioperative antibiotic prophylaxis, nasogastric or orogastric tube placement, sequential compression devices, examination under anesthesia, placement of Foley catheter, and uterine manipulator [7].

Modified nerve-sparing radical hysterectomy, in the context of our chart review, was defined as hysterectomy involving full dissection of the paravesical, pararectal, and recto-vaginal spaces and obturator spaces, isolation and ligation of the uterine arteries at their origins, dissection of the tunnels of Wertheim bilaterally with unroofing and skeletonizing of the ureters, and removal of at least half of the uterosacral ligaments, cardinal ligaments, and a portion of the paracolpos. In this group of patients, endometriotic implants were present in at 
least some, if not all, of these locations [8].

The surgical technique was as follows: abdominal entry was performed with Veress needle and needle mapping [7]. Four trocar ports were placed (10 $\mathrm{mm}$ umbilical port, $5 \mathrm{~mm}$ suprapubic, and two $5 \mathrm{~mm}$ ports at the lower abdominal quadrants) [9]. Adhesiolysis was performed when necessary to restore anatomy for placement of the ports. [8] Thorough exploration of the abdomen and pelvis was performed to assess the extent of endometriosis, rule out any possible malignant disease, and evaluate the feasibility of the intended procedure.

Before the hysterectomy was started, any pelvic pathology and extrapelvic endometriosis and adhesions were treated to try to return the anatomy to "normal" as much as possible with use of either CO2 laser, PlasmaJet, laparoscopic scissor, or blunt dissection. Radical hysterectomy was then started with sharp anteversion of the uterus and development of the rectovaginal space by sharp and blunt dissection. Next, the retroperitoneum was opened by incising lateral and parallel to the infundibulopelvic ligaments. The round ligaments were isolated, divided, and ligated close to the pelvic wall. The peritoneum overlying the vesicouterine fold was incised, and the bladder was mobilized using a combination of blunt and sharp dissection. The pararectal and paravesical spaces were opened. The utero-ovarian ligaments were then isolated, divided, and ligated.

The ureters were dissected free from the medial leaf of the peritoneum. Extensive bilateral ureterolysis was required and encompassed dissection from the pelvic brim to the bladder as well as exploration of the ureteric canal. When the crossover of the uterine artery was reached, and the artery was isolated at its origin, it was divided and ligated. The uterine artery pedicle was dissected anteriorly over the ureter. The superior vesical arteries were preserved, if possible. Care was taken to avoid trauma to the adjacent viscera.

The peritoneum across the cul-de-sac was further divided, and the rectovaginal space was further developed. At least half of the uterosacral ligament was excised, and in some cases, the ligaments were ligated at their point of origin. The anterior and posterior colpotomies were performed using a moistened sponge stick or right angle retractor placed in the anterior and posterior fornix.

A significant portion of the posterior wall of the vagina and rectovaginal septum was removed where endometriosis was involved. The uterus, cervix, and a portion of vagina were thus amputated and removed vaginally. The vaginal vault was closed with running suture. During the entire procedure, care was taken to avoid any trauma to the pelvic nervous system.

Patients were discharged to home on the same day of surgery once they met recovery room criteria, such as tolerating oral intake, ambulating, and voiding freely. Out of our 52 patients, 11 were admitted overnight for pain control or observation. Only one patient, who underwent bowel resection, was admitted for three days until she was able to tolerate oral intake.

\section{Results}

A total of 52 patients underwent laparoscopic modified nerve-sparing radical hysterectomy for endometriosis between October 2006 and September 2013. Six of these patients underwent robot-assisted laparoscopic radical hysterectomy.

The most common preoperative symptom was chronic pelvic and abdominal pain. All patients had a history of extensive endometriosis with failed medical treatments that caused significant recurrent symptoms limiting their quality of life. Out of 52 patients, 33 had at least one prior surgery (range: 1-5) for the treatment of endometriosis. 


\section{Cureus}

The mean patient age was 44 years (range 32-55). All patients were found to have Stage IV endometriosis. The mean hospital stay was one day (range: 1-3). Adjunctive procedures, including salpingo-oophorectomy, appendectomy, disc excision of the bowel and ureteroneocystotomy, enterocele repair, and in one case, bowel resection, were performed as indicated. Of note, nine patients had a history of unilateral oophorectomy prior to surgery. At the time of modified radical hysterectomy, 14 patients underwent bilateral oophorectomy, nine patients underwent unilateral oophorectomy, and two patients underwent removal of ovarian remnant.

\section{Patient characteristics}

Age

Range 32-55

Prior surgery for treatment of endometriosis

33 out of 52

Number of surgeries for treatment of endometriosis

Range 0-5 surgeries

Prior unilateral or bilateral oophorectomy 9 unilateral oophorectomies

Unilateral oophorectomy at time of hysterectomy 9

Bilateral oophorectomy at time of hysterectomy

Removal of ovarian remnant at time of hysterectomy

Postoperative complications 3 (urinary retention, vaginal cuff abscess, infection of ureteral stent)

Length of hospital stay

Range 1-5 days

Follow-up

Range 1-65 months

\section{TABLE 1: Results}

Postoperative complications included one case of urinary retention, one vaginal cuff abscess, and one infected ureteral stent. All patients reported dramatic pain relief, and there were no reports of symptom recurrence on follow-up which was, on average, 32 months (range: 1-65).

\section{Discussion}

Minimally invasive surgery has revolutionized modern day surgery.[10] Despite advances in the field of minimally invasive surgery, a recent large cross-sectional study of nationwide hysterectomy rates found that among the almost 600,000 hysterectomies, $14 \%$ of them were performed laparoscopically. The majority of hysterectomies are still done by laparotomy. The general trend, however, is that more and more hysterectomies are performed by laparoscopy [11].

Radical surgery may be needed in patients with severe endometriosis, especially after failed medical therapies or conservative surgeries. A number of studies focusing on the feasibility of bowel and bladder resection, with or without hysterectomy, by either laparotomy or 
laparoscopy for deep infiltrating endometriosis, showed acceptable morbidity and improvement of pelvic pain and fertility outcome [11, 18-21]. Fedele, et al. reported the only study comparing the use of modified radical hysterectomy versus extrafascial hysterectomy via laparotomy as extirpative treatment of such conditions. [6] The mean follow-up of the extrafascial hysterectomy was $68.9+/-21.1$ months with $31 \%$ of women in this group reporting recurrent pain symptoms between six and 18 months of surgery. The mean follow-up of the radical hysterectomy group was $31.3+/-8.7$ months with no patients reporting recurrent pain symptoms or requiring further surgery.

Since the first laparoscopic radical hysterectomy was reported by Nezhat, et al. [12-16] in 1989 for the treatment of cervical cancer, the feasibility and efficacy of this procedure have been supported in both minimally invasive gynecology and gynecologic oncology literature [24-26]. The use of such technique for complete excision of severe endometriosis has not been reported in the literature. This case series represents the first such report of laparoscopic radical hysterectomy for such an indication.

Women who undergo ovarian preservation surgery have a higher recurrence rate than those who undergo radical surgical treatment. The recurrence rate of endometriosis after radical surgical treatment reported is approximately 3\% [27-28]. All patients in this study, some with long-term follow-up (average follow-up 32 months with a range of 1-65 months), experienced significant pain relief after hysterectomy. There was no difference noted between symptom recurrences in the group of patients with bilateral oophorectomy versus those with ovarian conservation, in contrast to prior studies. Of note, nine patients had a history of unilateral oophorectomy prior to surgery. At the time of modified radical hysterectomy, 14 patients underwent bilateral oophorectomy, nine patients underwent unilateral oophorectomy, and two patients underwent removal of ovarian remnant.

Adjunctive procedures, such as disc excision of the bowel and ureteroneocystotomy, were needed in certain patients to achieve complete excision of endometriotic lesions. The absence of intraoperative complications and low rate of postoperative complications in this series, however, is consistent with reported complication rates in the literature for laparoscopic radical hysterectomy for malignancy [26, 29-30]. This case series further supports the use of extirpative surgical treatment for patients with severe endometriosis causing significant lifestyle-limiting pelvic pain that is resistant to medical and conservative surgical treatments. Limitations to this series include its retrospective nature and small sample size. It is possible that more recurrences could occur if a larger sample size were involved.

In some cases, hysterectomy and full debulking of disease can be beneficial. With extensive and deeply infiltrating endometriosis, this case series has demonstrated that it is feasible and safe with acceptable outcomes. The advantages of minimally invasive surgery can thus be offered even the most severe cases of disease.

\section{Conclusions}

In cases of severe endometriosis, the use of laparoscopic modified nerve-sparing radical hysterectomy is a feasible and effective method for achieving long-term improvement in pain symptoms. There was no difference noted between symptom recurrences in the group of patients with bilateral salpingo-oophorectomy versus those with ovarian conservation, in contrast to prior studies. Of note, 14 patients underwent bilateral oophorectomy, nine patients underwent unilateral oophorectomy, and two patients underwent removal of ovarian remnant.

\section{Additional Information}




\section{Disclosures}

Human subjects: All authors have confirmed that this study did not involve human participants or tissue. Animal subjects: All authors have confirmed that this study did not involve animal subjects or tissue. Conflicts of interest: In compliance with the ICMJE uniform disclosure form, all authors declare the following: Payment/services info: All authors have declared that no financial support was received from any organization for the submitted work. Financial relationships: We declare(s) a grant from Johnson \& Johnson. We declare(s) a grant from Storz. We declare(s) a grant from Intuitive Surgical. We declare(s) a grant from Stryker. Other relationships: All authors have declared that there are no other relationships or activities that could appear to have influenced the submitted work.

\section{References}

1. Burney RO, Giudice LC: Endometriosis. Nezhat's Video Assisted Laparoscopy and Hysteroscopy. Nezhat C, Nezhat F, Nezhat C (ed): Cambridge Press, New York; 2013. 252.

2. Schenken RS. Malinak LR: Reoperation after initial treatment of endometriosis with conservative surgery. Am J Obstet Gynecol. 1978, 131:416-24.

3. Nezhat C, Nezhat F, Pennington E: Laparoscopic treatment of infiltrative rectosigmoid colon and rectovaginal septum endometriosis by the technique of videolaparoscopy and the $\mathrm{CO} 2$ laser. Br J Obstet Gynaecol. 1992, 49:664-7.

4. Nezhat C, Hajhosseini B, King LP: Laparoscopic management of bowel endometriosis: Predictors of severe disease and recurrence. JSLS. Oct-Dec (4):. 2011, 15:431-8. 10.4293/108680811X13176785203752

5. Veeraswamy A, Lewis M, Mann A, Kotikela S, Hajhosseini B, Nezhat C: Extragenital endometriosis. Clin Obstet Gynecol. 2010, 53:449-466. 10.1097/GRF.0b013e3181e0ea6e

6. Gany R, Clayton R, Hawe J: The effect of endometriosis and its radical laparoscopic excision on quality of life indicators. BJOG. 2000, 107:44-54.

7. King LP, Nezhat C, Nezhat F, Alkatout I, Mettler L, Ferland R, Nezhat C: Laparoscopic Access. Nezhat's Video Assisted Laparoscopy and Hysteroscopy. Cambridge Press, New York; 2013. 42.

8. Nezhat C, Buescher E, Paka C, Nezhat C, Nezhat F: Video-assisted laparoscopic treatment of endometriosis. Nezhat's Video Assisted Laparoscopy and Hysteroscopy. Nezhat C, Nezhat F, Nezhat C (ed): Cambridge Press, New York; 2013. 265.

9. Buescher E, Schipper E, Nezhat C: Laparoscopic equipment and operating room setup. Nezhat's Video Assisted Laparoscopy and Hysteroscopy. Nezhat C, Nezhat F, Nezhat C (ed): Cambridge Press, New York; 2013. 35.

10. Kelley WE: The evolution of laparoscopy and the revolution in surgery in the decade of the 1990s. JSLS. 2008, 12:351-7.

11. Turner LC, Shepherd JP, Wang L, Bunker CH, Lowder JL: Hysterectomy surgical trends: A more accurate depiction of the last decade?. Am J Obstet Gynecol. 2013, 208:277.

10.1016/j.ajog.2013.01.022

12. Nezhat C: Baltimore; 1989.

13. Nezhat C, Nezhat F: Videolaseroscopy for the treatment of upper, mid, and lower peritoneal cavity pathology. Presented at Annual Meeting of American Association of Gynecologic Laparoscopists (AAGL). November, 1990,

14. Nezhat CR, Nezhat FR, Silfen SL: Videolaseroscopy. The CO2 laser for advanced operative laparoscopy. Obstet Gynecol Clin North Am. 1991, 18:585-604.

15. Nezhat, C: Laparoscopic Radical Hysterectomy.

16. Nezhat, CR, Burrell MO, Nezhat FR, Benigno BB, Welander CE: Laparoscopic radical hysterectomy with paraaortic and pelvic node dissection. Am J Obstet Gynecol. 1992, 166:8645 .

17. Wright JD, Ananth CV, Lewin SN, et al.: Robotically assisted vs laparoscopic hysterectomy among women with benign gynecologic disease. JAMA. 2013, 309:689-98.

10.1001/jama.2013.186

18. Coronado C, Franklin RR, Lotze EC, Bailey HR, Valdés CT: Surgical treatment of symptomatic colorectal endometriosis. Fertil Steril. 1990, 53:411-6.

19. Nezhat C, Nezhat F, Pennington E: Laparoscopic treatment of infiltrative rectosigmoid colon 
and rectovaginal septum endometriosis by the technique of videolaparoscopy and the CO2 laser. Br J Obstet Gynecol. 1992, 99:664-7.

20. Bailey HR, Ott MT, Hartendorp P: Aggressive surgical management for advanced colorectal endometriosis. Dis Colon Rectum. 1994, 37:747-53.

21. Dupree HJ, Senagore AJ, Delaney CP, Manrcello PW, Brady KM, Falcone T: Laparoscopic resection of deep pelvic endometriosis with rectosigmoid involvement. J Am Coll Surg. 2002, 195:754-8.

22. Comiter CV: Endometriosis of the urinary tract. Urol Clin North Am. 2002, 29:625-35.

23. Darai E, Thomassin, I, Barranger E, Detchev R, Cortez A, Houry S, Bazot M: Feasibility and clinical outcome of laparoscopic colorectal resection for endometriosis. Am J Obstet Gynecol. 2005, 192:394-400.

24. Obemiair A, Ginbey P, McCartney AJ: Feasibility and safety of total laparoscopic radical hysterectomy. J Am Assoc Gynecol Laparosc. 2003, 10:345-9.

25. Malzoni M, Tinelli R, Cosentino F, Perone C, Vicario V: Feasibility, morbidity, and safety of total laparoscopic radical hysterectomy with lymphadenectomy: Our experience. J Minim Invasive Gynecol. 2007, 14:584-90.

26. Abu-Rustum NR, Gemignani ML, Moore K, Sonoda Y, Venkatraman E, Brown C, Poynor E, Chi DS, Barakat RR: Total laparoscopic radical hysterectomy with pelvic lymphadenectomy using the argon-beam coagulator: Pilot data and comparison to laparotomy. Gynecol Oncol. 2003, 91:402-9; Erratum in Gynecol Oncol. 2004 Apr;93(1):275.

27. Zakashansky K, Chuang L, Gretz H, Nagarsheth NP, Rahaman J, Nezhat FR: A case-controlled study of total laparoscopic radical hysterectomy with pelvic lymphadenectomy versus radical abdominal hysterectomy in a fellowship training program. Int J Gynecol Cancer. 2007, 17:1075-82.

28. Frumovitz M, dos Reis R, Sun CC, Milam MR, Bevers MW, Brown J, Slomovitz BM, Ramirez PT: Comparison of total laparoscopic and abdominal radical hysterectomy for patients with earlystage cervical cancer. Obstet Gynecol. 2007, 110:96-102.

29. Namnoum AB, Hickman TN, Goodman SB, Gehlbach DL, Rock JA: Incidence of symptom recurrence after hysterectomy for endometriosis. Fertil Steril. 1995, 64:898-902.

30. Matorras R, Elorriaga MA, Pijoan JI, Ramón O, Rodríguez-Escudero FJ: Recurrence of endometriosis in women with bilateral adnexectomy (with or without total hysterectomy) who received hormone replacement therapy. Fertil Steril. 2002, 77:303-308. 